\title{
Difference of the Nuclear Green Light Intensity between Papillary Carcinoma Cells Showing Clear Nuclei and Non-neoplastic Follicular Epithelia in Papillary Thyroid Carcinoma
}

\author{
Hyekyung Lee · Tae Hwa Baek ${ }^{1}$ \\ Meeja Park · Seung Yun Lee \\ Hyun Jin Son · Dong Wook Kang \\ Joo Heon Kim · Soo Young Kim² \\ Department of Pathology, Eulji University Hospital, \\ Eulji University School of Medicine, Daejeon; \\ ${ }^{1}$ Medical Examiner's Office, National Forensic \\ Service, Wonju; '2Department of Occupational \\ Medicine, Eulji University Hospital, Eulji University \\ School of Medicine, Daejeon, Korea \\ Received: March 11, 2016 \\ Revised: May 3, 2016 \\ Accepted: May 19, 2016 \\ Corresponding Author \\ Soo Young Kim, MD \\ Department of Pathology, Eulji University Hospital, \\ 95 Dunsanseo-ro, Seo-gu, Daejeon 35233, Korea \\ Tel: +82-42-611-3782 \\ Fax: $+82-42-611-3776$ \\ E-mail: kimsooy@eulji.ac.kr
}

\begin{abstract}
Background: There is subjective disagreement regarding nuclear clearing in papillary thyroid carcinoma. In this study, using digital instruments, we were able to quantify many ambiguous pathologic features and use numeric data to express our findings. Methods: We examined 30 papillary thyroid carcinomas. For each case, we selected representative cancer cells showing clear nuclei and surrounding non-neoplastic follicular epithelial cells and evaluated objective values of green light intensity (GLI) for quantitative analysis of nuclear clearing in papillary thyroid carcinoma. Results: From 16,274 GLI values from 600 cancer cell nuclei and 13,752 GLI values from 596 nonneoplastic follicular epithelial nuclei, we found a high correlation of $94.9 \%$ between GLI and clear nuclei. GLI between the cancer group showing clear nuclei and non-neoplastic follicular epithelia was statistically significant. The overall average level of GLI in the cancer group was over two times higher than the non-neoplastic group despite a wide range of GLI. On a polygonal line graph, there was a fluctuating unique difference between both the cancer and non-neoplastic groups in each patient, which was comparable to the microscopic findings. Conclusions: Nuclear GLI could be a useful factor for discriminating between carcinoma cells showing clear nuclei and non-neoplastic follicular epithelia in papillary thyroid carcinoma.
\end{abstract}

Key Words: Image processing, computer-assisted; Light intensity; Cell nucleus; Thyroid cancer, papillary
In the diagnosis of papillary thyroid carcinoma, nuclear characteristics are important. Among the nuclear triad composed of clear nuclei, nuclear grooves and pseudoinclusions, nuclear clearing is the most subjective and most ambiguous finding. ${ }^{1}$ Currently, with the use of digital instruments, we are able to quantify many ambiguous pathologic features and express our findings with numeric data. ${ }^{2-6}$ Based on a previous study that described the meaningful data of nuclear green light intensity (GLI) in neuroendocrine tumors, ${ }^{7}$ we hypothesized that the nuclear clearing of papillary thyroid carcinoma could be also expressed using GLI because both tumors share similar nuclear features with relatively homogeneous nuclei without irregular chromatin clumping. We measured the GLI of cancer cells showing clear nuclei and surrounding non-neoplastic follicular epithelial nuclei in papillary thyroid carcinoma patients and evaluated the significance of GLI as a discriminator between the two groups.

\section{MATERIALS AND METHODS}

A total of 30 thyroidectomy specimens with papillary carcinoma were retrospectively selected from the Department of Pathology and Laboratory Medicine archives of the Eulji University Hospital. We cut formalin-fixed, paraffin-embedded tissues

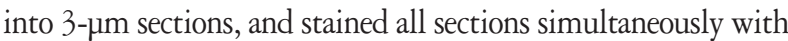
hematoxylin and eosin using an automatic slide staining machine under standard conditions. We reviewed the slides on an Olympus BX51 microscope (Olympus Corp., Tokyo, Japan) with $\times 40$ magnification of the apochromatic objective lens and a 0.85 numeric aperture. We maintained the same light source conditions throughout the analysis. Clear nuclei were determined by two pathologists (H.L. and M.P.). We captured digital images $(1,360 \times 1,024$ pixels) of typical areas showing cancer cells with clear nuclei and surrounding non-neoplastic nuclei by using an Olympus DP71 digital camera (Olympus Corp.) and saved the 
Table 1. The number of GLI of cancer cells and non-neoplastic cells in 30 papillary thyroid carcinoma patients

\begin{tabular}{|c|c|c|c|c|}
\hline Patient No. & No. of cancer cells & No. of GLI of cancer & No. of non-neoplastic cells & No. of GLI of non-neoplastic cells \\
\hline 1 & 20 & 533 & 16 & 354 \\
\hline 2 & 20 & 542 & 20 & 470 \\
\hline 3 & 20 & 558 & 20 & 435 \\
\hline 4 & 20 & 485 & 20 & 443 \\
\hline 5 & 20 & 463 & 20 & 417 \\
\hline 6 & 20 & 372 & 20 & 377 \\
\hline 7 & 20 & 483 & 20 & 467 \\
\hline 8 & 20 & 433 & 20 & 373 \\
\hline 9 & 20 & 466 & 20 & 401 \\
\hline 10 & 20 & 497 & 20 & 342 \\
\hline 11 & 20 & 476 & 20 & 367 \\
\hline 12 & 20 & 423 & 20 & 415 \\
\hline 13 & 20 & 442 & 20 & 345 \\
\hline 14 & 20 & 452 & 20 & 380 \\
\hline 15 & 20 & 449 & 20 & 427 \\
\hline 16 & 20 & 546 & 20 & 434 \\
\hline 17 & 20 & 496 & 20 & 424 \\
\hline 18 & 20 & 635 & 20 & 428 \\
\hline 19 & 20 & 519 & 20 & 459 \\
\hline 20 & 20 & 537 & 20 & 455 \\
\hline 21 & 20 & 547 & 20 & 417 \\
\hline 22 & 20 & 521 & 20 & 414 \\
\hline 23 & 20 & 508 & 20 & 437 \\
\hline 24 & 20 & 575 & 20 & 425 \\
\hline 25 & 20 & 750 & 20 & 605 \\
\hline 26 & 20 & 623 & 20 & 662 \\
\hline 27 & 20 & 798 & 20 & 604 \\
\hline 28 & 20 & 760 & 20 & 692 \\
\hline 29 & 20 & 630 & 20 & 625 \\
\hline 30 & 20 & 755 & 20 & 658 \\
\hline Total & 600 & 16,274 & 596 & 13,752 \\
\hline
\end{tabular}

GLI, green light intensity.

images in Tagged Image File Format format. We reviewed each digital image and removed any image that did not fulfill the minimum requirements (bad nuclear mask, out-of-focus image, pyknotic nucleus). For each case, we selected 20 representative cancer cells with clear nuclei and 20 surrounding non-neoplastic follicular epithelial nuclei except for case 1. For each cell, we drew line profiles using computerized image analyzer (Image Pro program Plus ver. 6.5, Media Cybertics Co., Silver Spring, MD, USA) and obtained graph files and Excel data on GLIs of consecutive pixels along the line profiles. For comparison between the two groups, statistical analysis was performed with a nonparametric Mann-Whitney $\mathrm{U}$ test and $\mathrm{p}<.05$ was considered significant.

\section{RESULTS}

A total of 600 cancer cell nuclei and 596 non-neoplastic fol-

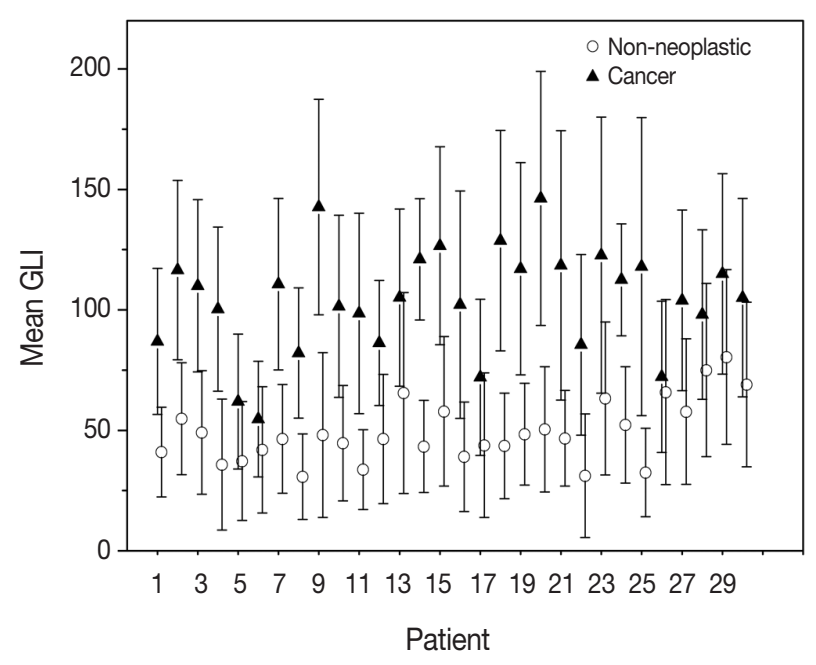

Fig. 1. Plot box graph of green light intensity (GLI) of cancer cells and non-neoplastic cells in 30 papillary thyroid carcinoma patients. Error bar, 5th and 95th percentiles; line, central 50th percentile; triangle for cancer, circle for non-neoplastic group, mean GLI. 
licular epithelial nuclei were selected from 30 papillary thyroid carcinoma patients. The 16,274 GLI values from 600 cancer cell nuclei and 13,752 GLI values from 596 non-neoplastic follicular epithelial nuclei were studied (Table 1, Fig. 1). We sought to determine whether GLI could be used to identify clear nuclei of the cancer group by comparison with the non-neoplastic group. There was a high correlation of $94.9 \%$ between GLI and clear nuclei on the receiver operating characteristic (ROC) curve (Fig. 2). There was a significant increase in GLI in the cancer group (GLI median, standard deviation); with values of 51, 20 for the non-neoplastic group compared to 105, 29 for the cancer group, which was statistically significant (Fig. 3). On a po-

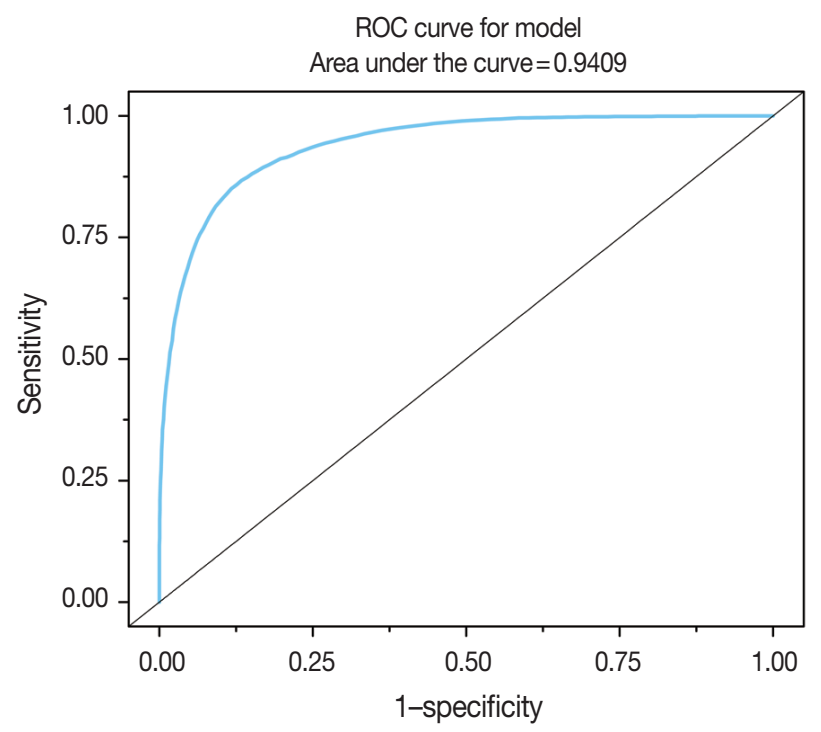

Fig. 2. High correlation of green light intensity with nuclear clearing in receiver operating characteristic curve (ROC).

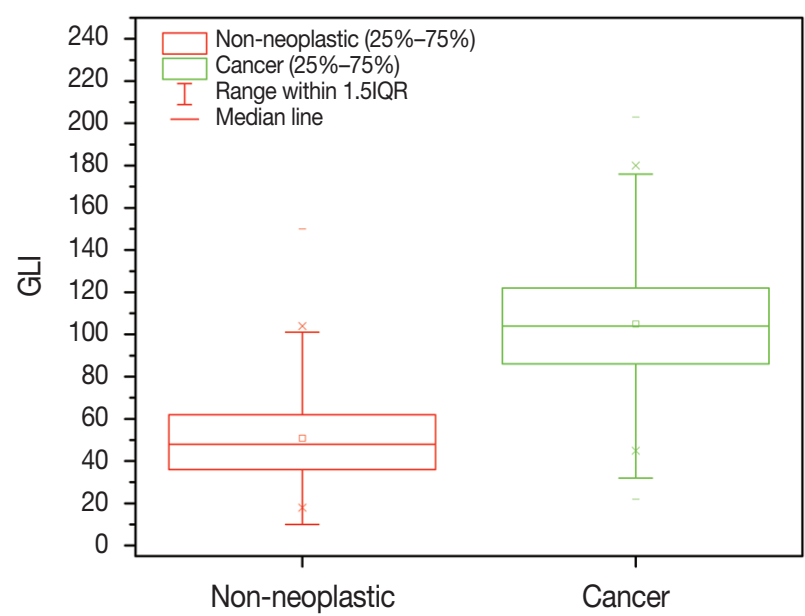

Fig. 3. Comparison of green light intensity (GLI) between papillary thyroid carcinoma showing clear nuclei and surrounding non-neoplastic follicular epithelial cells. lygonal line graph of an equal number of GLI from the cancer group and non-neoplastic group in each patient, there was a fluctuating difference between each patient, but there was a higher difference in the cancer group (Fig. 4). The microscopic findings of cases 9, 14, and 25 who showed more GLI difference between the two groups revealed more clear nuclei in cancer cells (Fig. 5A), whereas cases 5, 6, and 26 showed less GLI difference between the two groups with less clear nuclei in cancer cells (Fig. 5B). Each patient showed unique identifiable patterns in both the cancer and non-neoplastic groups.
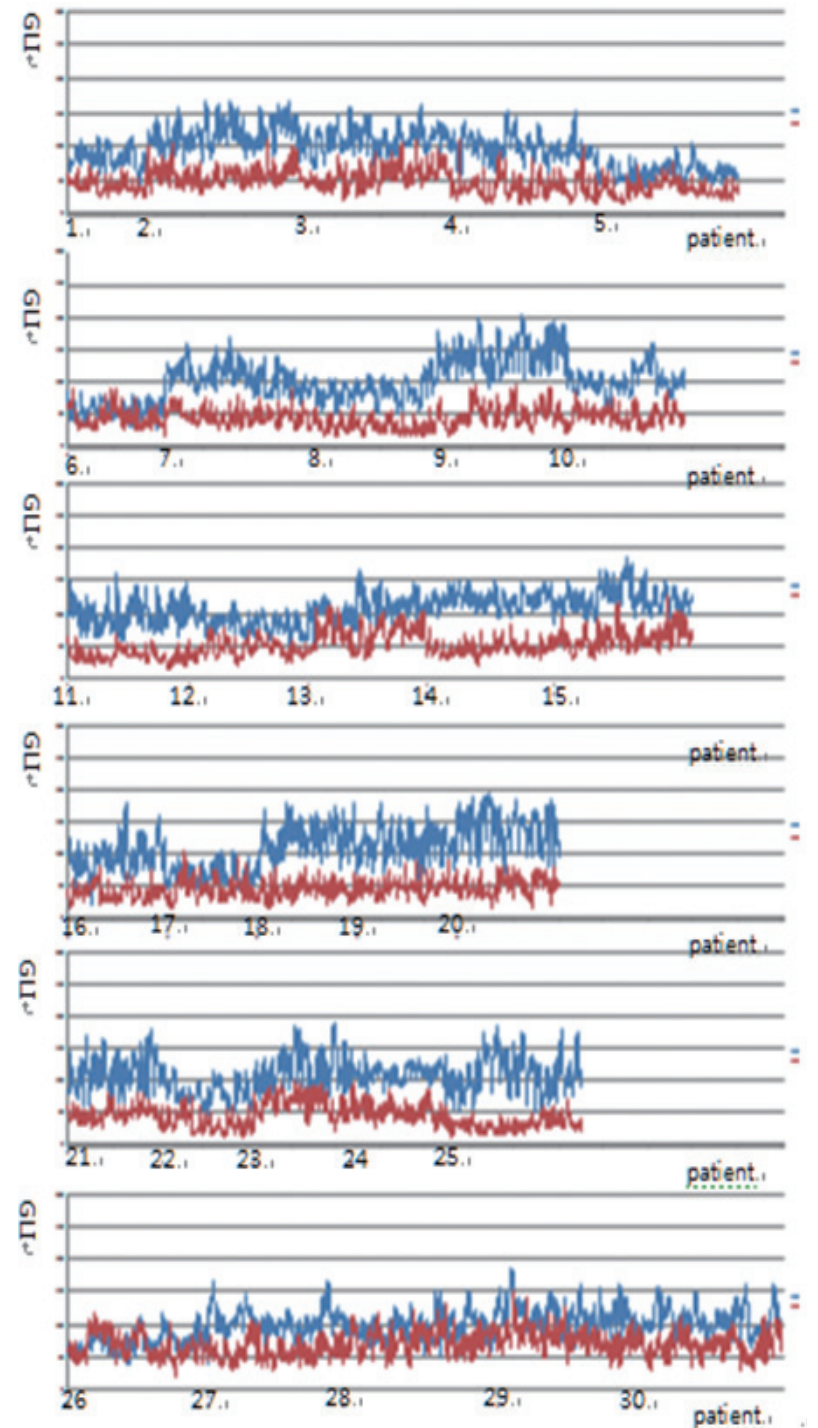

Fig. 4. Fluctuating difference of green light intensity (GLI) in both cancer and non-neoplastic groups between each patient on polygonal line graph (red, non-neoplastic cells; blue, cancer). 

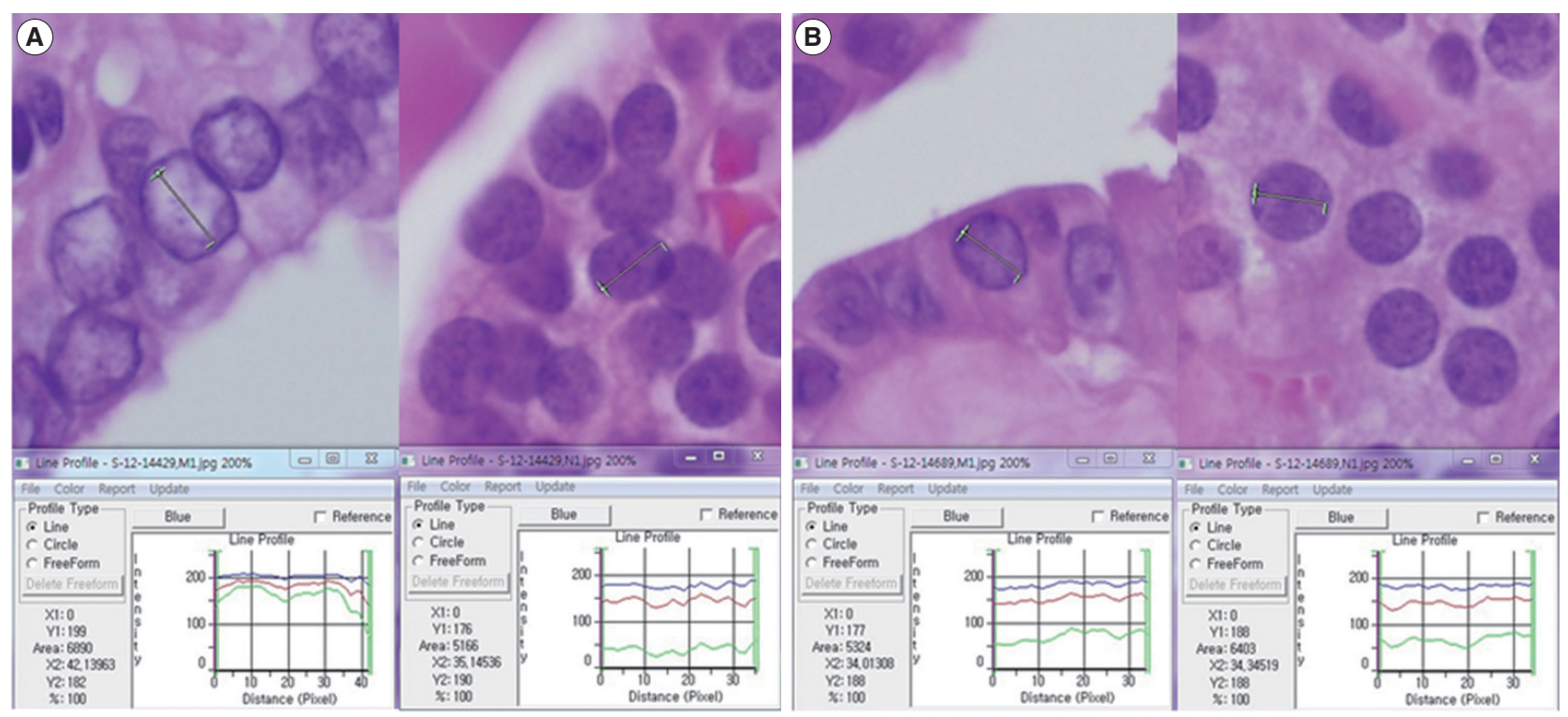

Fig. 5. (A) Comparison of green light intensity (GLI) of cancer cells and non-neoplastic cells in case 25, which shows a large difference (left, cancer; right, non-neoplastic cells). (B) Comparison of GLI of cancer cells and non-neoplastic cells in case 26, where there is no significant difference (left, cancer; right, non-neoplastic cells). Note the similarity in red, green, and blue light intensities of non-neoplastic follicular epithelial cells in panel $A$ and $B$.

\section{DISCUSSION}

Nuclear examination of cells is important in pathology. Several characteristic nuclear features of clinical significance have been described. Hyperchromatism is an expression of increased DNA content due to cellular proliferation and mitosis is an expression of rapid cellular division. Coffee bean-like nuclear grooves, ground glass nuclei, and nuclear pseudoinclusions are meaningful in the diagnosis for specific diseases such as papillary thyroid carcinoma. Among those nuclear features, pseudoinclusions or grooves are relatively objective findings with either presence or absence documented. However, some features such as clear nuclei, vesicular nuclei, and homogenized nuclei are relatively subjective findings and their diagnosis is subject to interobserver variability. Many pathologists have been trying to minimize interobserver variability for some time. Currently, by using a more developed digital imaging system, we are able to translate subjective and ambiguous pathologic findings into objective data. With these data, we can reduce interobserver variability and introduce a new era of data based research. Papillary thyroid carcinoma is a well-known tumor in the sense that appropriate nuclear features are essential for making the diagnosis. Among these diagnostic nuclear features, clear nuclei are relatively subjective and shows significant interobserver variability. Several questions regarding clear nuclei remain, including the following. What degree of clearance is enough for the diagnosis of clear nuclei of papillary carcinoma? Is the level of clearing the same in usual papillary thyroid carcinomas? Is there any recommended method to measure the clearing? Unfortunately, the recommended and standard method for measuring nuclear clearing is not yet known. When we considered these issues, we realized the necessity of measurable data regarding the degree and level of nuclear clearing. In addition to quantitative studies regarding nuclear morphometry characteristics such as variation in nuclear size, irregularity in nuclear contours, and chromatin texture ${ }^{3-7}$ red, green, and blue color charts have also been used to calibrate digital graphic devices. ${ }^{8,9}$ A recent study described red, green, and blue light intensities for nuclear chromatin pattern of neuroendocrine cells and stressed the importance of GLI. ${ }^{7}$ We attempted to answer the above questions by quantitatively measuring objective GLI values. In the materials and methods section, we questioned whether single line profile can be used instead of different orientations $\left(0^{\circ}, 45^{\circ}, 90^{\circ}\right.$, and $\left.135^{\circ}\right)$. In a short preliminary study (unpublished data) we could not find big differences between those groups and in practice, it was not easy to draw a line profile of different angles because we had to avoid nuclear grooves and pseudoinclusions. In the results analysis, by comparing the GLI between the cancer group with clear nuclei and the non-neoplastic internal control group, we were able to reduce the possible errors that affect digital image analysis, namely specimen thickness, staining, and interference due to the brightness around the line profiles. Fig. 2 shows a high con- 
cordance of $94.9 \%$ between the carcinoma groups and GLI in the ROC curve study. Fig. 3 shows the difference in GLI between the cancer group showing clear nuclei and the non-neoplastic group, which was statistically significant. Based on these results, we suggest that the presence of clear nuclei is highly correlated with GLI and that GLI could be a meaningful discriminator between cancer and non-neoplastic groups. Fig. 4 shows the fluctuating difference of GLI in both cancer and non-neoplastic groups in each case, with an even higher difference in the cancer group. The higher fluctuation in the cancer group seems to be related to the higher standard deviation described in Table 1. In cases 5, 6, and 26, the GLI difference between the cancer and non-neoplastic groups was not significant; however in cases 9 , 14 , and 25 , the difference between the two groups was significant. These differences in GLI were easily identified by microscopic examination for clear nuclei (Fig. 5). Based on such differences in GLI compared with the microscopic findings in each case, we were able to answer the second question as follows: the level of clearing is not the same in all thyroid papillary carcinomas. When we considered that nuclear clearing can be related to nuclear microfilament localization or hypochromatism in tumor progression ${ }^{10,11}$ with the wide range of light intensity, the nuclear clearing could be an expression of gradual tumor progression rather than a sudden onset phenomenon. The pathogenesis of a clear nucleus is not completely understood. The effects of thyroid hormone on $\mathrm{Na} / \mathrm{K}$-ATPase activity have been studied and the functions of the Na/K-ATPase of the nuclear envelope in ion transport and chromatin modulation have been described. ${ }^{12-15}$ Therefore, the characteristic nuclear triad of papillary thyroid carcinoma, which consists of nuclear grooves, pseudoinclusions, and clearing, can be secondary findings due to an abnormal Na/K-ATPase affected by abnormal thyroid hormone in papillary thyroid carcinoma. Because of the wide range of GLI, we could not generate a diagnostically helpful cut-off value or function for the discrimination between the cancer group showing clear nuclei and non-neoplastic group. However, with over 30,000 GLI data points, we believe that an average level of GLI at least two times higher in the cancer group (104.9) than the non-neoplastic group (50.8) is meaningful. For example, in thyroid tissue section showing big difference over two times of GLI of each cell, although we can't definitely discriminate between cancer cells showing clear nuclei and normal cells only by GLI values, we could consider the possibility of papillary carcinoma in the tissue section. Moreover we can apply these results in cytology, where automatic screening is relatively popular. However, there are still many problems to be solved, such as common nuclear clearing in autoimmune thyroiditis and proper quality control and adjustment for different samples. Recently, Louis et al..$^{16,17}$ defined computational pathology as an approach to extract clinically actionable knowledge from variable data, including digital images, and suggested that computational pathology should serve as a hub for data-related research in modern health care systems. By using newly developed information and technology tools, we can review and reanalyze our traditional pathologic findings and with those efforts, we can expand our understanding of diseases and obtain more objective and reproducible methods of diagnosis. In conclusion, quantitative analysis of the nuclear GLI for the discrimination between cancer cells showing clear nuclei and non-neoplastic follicular epithelial cells in papillary thyroid carcinoma revealed meaningful results comparable to traditional microscopic findings. Nuclear GLI could be a useful factor for the discrimination between these two groups.

\section{Conflicts of Interest}

No potential conflict of interest relevant to this article was reported.

\section{REFERENCES}

1. LiVolsi VA. Papillary thyroid carcinoma: an update. Mod Pathol 2011; 24 Suppl 2: S1-9.

2. Neltner JH, Abner EL, Schmitt FA, et al. Digital pathology and image analysis for robust high-throughput quantitative assessment of Alzheimer disease neuropathologic changes. J Neuropathol Exp Neurol 2012; 71: 1075-85.

3. El Hallani S, Guillaud M, Korbelik J, Marginean EC. Evaluation of quantitative digital pathology in the assessment of Barrett eophagus-associated dysplasia. Am J Clin Pathol 2015; 144: 151-64.

4. Guillaud M, Zhang L, Poh C, Rosin MP, MacAulay C. Potential use of quantitative tissue phenotype to predict malignant risk for oral premalignant lesions. Cancer Res 2008; 68: 3099-107.

5. Huang W, Hennrick K, Drew S. A colorful future of quantitative pathology: validation of Vectra technology using chromogenic multiplexed immunohistochemistry and prostate tissue microarrays. Hum Pathol 2013; 44: 29-38.

6. Kayser K, Görtler J, Goldmann T, Vollmer E, Hufnagl P, Kayser G. Image standards in tissue-based diagnosis (diagnostic surgical pathology). Diagn Pathol 2008; 3: 17.

7. Park M, Baek T, Baek J, et al. Nuclear image analysis study of neuroendocrine tumors. Korean J Pathol 2012; 46: 38-41. 
8. Yagi Y. Color standardization and optimization in whole slide imaging. Diagn Pathol 2011; 6 Suppl 1: S15.

9. Bautista PA, Hashimoto N, Yagi Y. Color standardization in whole slide imaging using a color calibration slide. J Pathol Inform 2014; 5: 4 .

10. Johannessen JV, Gould VE, Jao W. The fine structure of human thyroid cancer. Hum Pathol 1978; 9: 385-400.

11. Fischer AH, Taysavang P, Weber CJ, Wilson KL. Nuclear envelope organization in papillary thyroid carcinoma. Histol Histopathol 2001; 16: 1-14.

12. Lin $\mathrm{MH}$, Akera $\mathrm{T}$. Increased $(\mathrm{Na}+, \mathrm{K}+)$-ATPase concentrations in various tissues of rats caused by thyroid hormone treatment. J Biol
Chem 1978; 253: 723-6.

13. Kamitani T, Ikeda U, Muto S, et al. Regulation of Na,K-ATPase gene expression by thyroid hormone in rat cardiocytes. Circ Res 1992; 71: 1457-64.

14. Liu Y, Levine B. Autosis and autophagic cell death: the dark side of autophagy. Cell Death Differ 2015; 22: 367-76.

15. Garner MH. Na,K-ATPase in the nuclear envelope regulates $\mathrm{Na}+$ : K+ gradients in hepatocyte nuclei. J Membr Biol 2002; 187: 97-115.

16. Louis DN, Gerber GK, Baron JM, et al. Computational pathology: an emerging definition. Arch Pathol Lab Med 2014; 138: 1133-8.

17. Louis DN, Feldman M, Carter AB, et al. Computational pathology: a path ahead. Arch Pathol Lab Med 2016; 140: 41-50. 